\title{
Evaluation of Anastomosis With ICG Fluorescence Method Using VISERA ELITE2 During Laparoscopic Colorectal Cancer Surgery
}

\author{
SHUNJIN RYU, KATSUHITO SUWA, TAKAHIRO KITAGAWA, MARIE AIZAWA, \\ TAKUROU USHIGOME, TOMOYOSHI OKAMOTO, KEN ETO and KATSUHIKO YANAGA \\ Department of Surgery, The Jikei University Hospital, Tokyo, Japan
}

\begin{abstract}
Aim: In colorectal cancer surgery, the efficacy of intestinal blood flow evaluation with the indocyanine green (ICG) fluorescence method using the VISERA ELITE2 system was investigated. Patients and Methods: Participants in this study comprised 50 patients who underwent elective laparoscopic colorectal cancer surgery at the Department of Surgery, the Jikei Daisan Hospital. With the ICG fluorescence method, whether it was necessary to change the intestinal transection line for anastomosis was evaluated. Results: For three cases of rectal cancer, the oral transection line determined from macroscopic observation was judged to offer insufficient blood flow according to the ICG fluorescence method. The transection line for anastomosis was changed according to fluorescence. None of these cases showed complications. Conclusion: The ICG fluorescence method may allow safe anastomosis in colorectal surgery for cancer.
\end{abstract}

In colorectal cancer surgery, lymph node dissection is performed by ligating and resecting the main artery feeding the tumor. Relative degeneration of intestinal blood flow (IBF) to the anastomotic site may contribute to anastomotic leakage. Indocyanine green (ICG) is a dye used in liver function testing which is excited when irradiated with nearinfrared light, and can be seen as fluorescence using a nearinfrared fluorescence camera. ICG has gradually been adapted to use in colorectal cancer surgery. Use of this method in preoperative colonic cancer tattooing (1), IBF evaluation of anastomosis to avoid leakage (2-6) and

Correspondence to: Shunjin Ryu, Department of Surgery, The Jikei University Hospital, 3-19-18, Nishishinbashi, Minato city, Tokyo 105-8461, Japan. Tel: +81 334331111, e-mail: systematic.ryu1121@gmail.com

Key Words: Indocyanine green fluorescence, anastomosis, colorectal cancer, intestinal blood flow. evaluation of intestinal lymph flow (7) has been reported. At our hospital, a near-infrared fluorescence camera (VISERA ELITE2 system; Olympus, Tokyo, Japan) has been adopted for use in colorectal surgery.

This study evaluated whether the ICG fluorescence method results in changes to the operative plan, such as decision making in the intestinal transection line for anastomosis.

\section{Patients and Methods}

Participants in this study comprised 50 patients who underwent elective laparoscopic colorectal cancer surgery between October 2017 and November 2018 at the Department of Surgery, the Jikei Daisan Hospital.

Each participant provided informed consent to participate in this study. Cases with surgery involving two anastomoses, such as double cancer, were excluded.

Outcome measurements were patient characteristics, surgical outcomes and whether the position of the planned colorectal transection line was changed after IBF evaluation using the ICG fluorescence method. Laparoscopic surgery was performed as usual with total mesorectal excision or complete mesocolic excision. The main artery feeding the tumor was ligated for lymph node dissection. We determined the intestinal transection line based on intestinal blood flow assessment depending on tactile and visual sensations to secure the transection margin in accordance with the Japanese Classification of Colorectal Carcinoma (8). In operations with transanal anastomosis (low anterior resection, sigmoidectomy, intersphincteric resection), IBF assessment with the ICG fluorescence method was performed for the oral-side of the intestine. On the other hand, in operations with ileocolostomy (ileocecal resection, right hemi-colectomy), IBF assessment was performed for the anal-side of the intestine. The intestines after the division of the mesentery were observed using a VISERA ELITE2 system (Olympus, Tokyo, Japan). ICG was injected intravenously at $0.1 \mathrm{mg} / \mathrm{kg}$. The necessity for changing the intestinal transection line for optimal anastomosis was evaluated using the ICG fluorescence method.

Results are expressed as mean values. This study was approved by the Research Ethics Committee of the Jikei University School of Medicine (Tokyo, Japan) (approval number: 29-049). 


\section{Results}

A total of 50 patients were analyzed in this study. Table I shows patient characteristics. Mean age was 70.5 years (range $=35-89$ years), with 27 men and 23 women. The majority of tumor were located in the colon $(29 / 50,58 \%)$. Preoperative intestinal obstruction was seen in six cases (12\%). Preoperative chemoradiotherapy with capecitabine was performed for two cases of lower rectal cancer. In 47 cases, the transection line determined by macroscopic observation was judged to provide sufficient blood flow with the ICG fluorescence method (Figure 1).

In one case of rectosigmoidal cancer (Figure 2), and two cases of lower rectal cancer (Figures 3 and 4), the oral transection line to form the anastomosis as determined by macroscopic observation was judged as providing insufficient blood flow with the ICG fluorescence method (Table II). The transection line to form the anastomosis was changed to a position providing good fluorescence. These cases showed no complications. In the operation with ileocolostomy (ileocecal resection, right hemi-colectomy), changes to the transection line with the ICG fluorescence method were not made. Post-operative complications were paralytic ileus, abdominal abscess, anastomotic leakage and stomal obstruction in one case each, and chylous ascites, and wound infection in two cases each (Table III). Postoperative complications defined as Clavien-Dindo grade III (9) or more occurred in one case, which was anastomotic leakage $(2 \%)$.

\section{Discussion}

Causes of anastomotic leakage are thought to include insufficient blood flow, incompleteness of the anastomosis itself, tension on the intestine around the anastomosis, distal obstruction and predisposing factors of the individual patient (10-12). The degree of completeness of the anastomosis itself can be checked with a leak test or intraoperative endoscopy in cases of transanal anastomosis, in addition to observation of the anastomosis during surgery. Assessment of tension on the intestine around the anastomosis was performed during the operation and mobilization of the intestine was added as necessary. If an anastomosis breaks down after being confirmed as complete and not under tension during surgery, the possibility of insufficient blood flow or a predisposing factor of the patient is highly likely. Patient predispositions include malnutrition and obstructive colitis due to intestinal obstruction (10-12). Although patient predispositions can be improved by preoperative management, appropriate assessments of the anastomosis and sometimes avoidance of anastomosis are needed if the predisposing condition of the patient remains a concern. Given such a situation, multiple factors affect anastomotic
Table I. Patient characteristics.

\begin{tabular}{lc}
\hline Characteristic & Value \\
\hline Age, years & \\
Median (range) & $70.5(35-89)$ \\
Gender, n (\%) & \\
Male & $27(54 \%)$ \\
Female & $23(46 \%)$ \\
Body mass index, kg/m ${ }^{2}$ & \\
Median (range) & \\
Preoperative factor, $\mathrm{n}(\%)$ & $(16.89-35.80)$ \\
Obstruction & $6(12 \%)$ \\
Therapy & $2(4 \%)$ \\
Location of cancer, $\mathrm{n}(\%)$ & \\
Cecum & $8(16 \%)$ \\
Ascending colon & $10(20 \%)$ \\
Transverse colon & $3(6 \%)$ \\
Descending colon & $1(2 \%)$ \\
Sigmoidal colon & $7(14 \%)$ \\
Rectosigmoidal colon & $7(14 \%)$ \\
Upper rectum & $4(8 \%)$ \\
Lower rectum & $10(20 \%)$ \\
Comorbidity, n $(\%)$ & \\
Angina pectoris & $2(4 \%)$ \\
Arrhythmia & $3(6 \%)$ \\
Hypertension & $22 \%)$ \\
Diabetes & $2(6 \%)$ \\
Respiratory disease & $11 \%)$ \\
Neurological disease & \\
Crohn's disease & \\
Other malignant disease & \\
\hline
\end{tabular}

leakage in a complicated manner, but the ICG fluorescence method may reduce the risk of adverse impacts due to insufficient blood flow. IBF is usually evaluated by observing bowel color, the presence or absence of peristalsis, the presence or absence of bleeding from the bowel resection stump, and arterial pulsations in the mesentery. However, these rely on the subjective interpretation of the surgeon and evaluation of bowel ischemia can be difficult in some patients. The ICG fluorescence method can make IBF evaluation more objective.

With evaluation by ICG fluorescence, IBF can be observed by injecting ICG from a peripheral vein. This can be highly useful from the perspectives of convenience, speed, and observation of a wide area. The VISERA ELITE2 system is a scope for laparoscopic surgery that allows a switch to fluorescence observation with a touch of a button without changing the light guide cable or adding another camera. This allows surgery and observation of fluorescence without interruption. In the PILLAR II study, IBF assessment with the ICG fluorescence method using PINPOINT (endoscopic fluorescence imaging camera; Stryker, Kalamazoo, MI, USA) reduced anastomotic leakage to $1.4 \%$ 

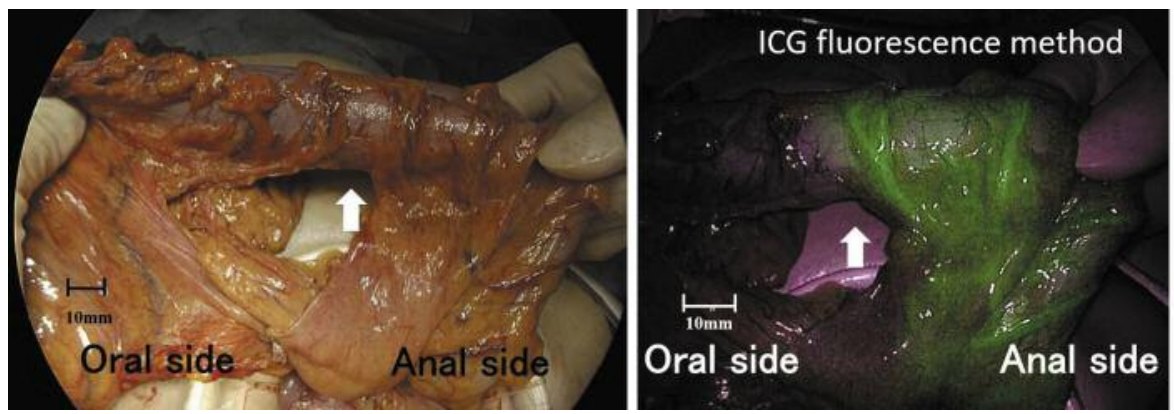

Figure 1. The patient in this case was a 69-year-old woman with ascending colon cancer. The white arrow indicates the anal-side intestinal transection line from blood flow assessment by tactile and visual sensation. This position was highly fluorescent under the indocyanine green (ICG) fluorescence method. The intestinal transection line for the anastomosis was left unchanged.
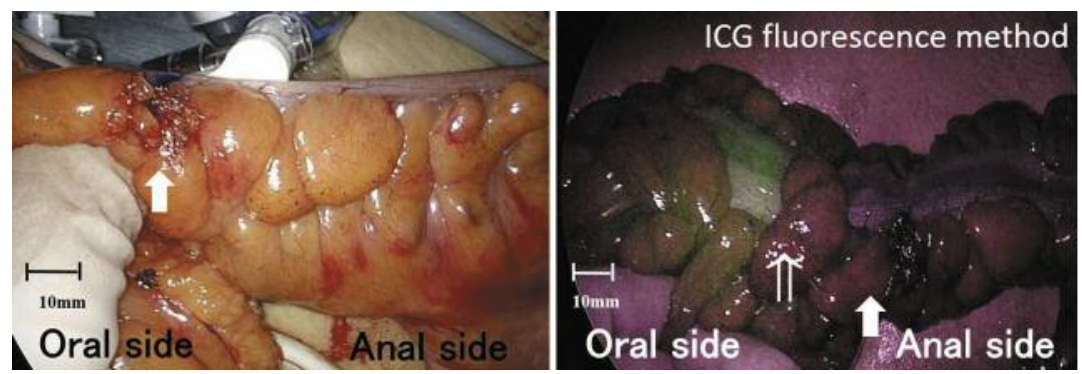

Figure 2. The patient in this case was a 54-year-old man with rectosigmoidal cancer. The white arrow indicates the oral-side intestinal transection line from blood flow assessment by tactile and visual sensation. This line did not show good fluorescence with the indocyanine green (ICG) fluorescence method. The intestinal transection line for anastomosis was changed to the position indicated by the open white arrow. The distance between positions was $3 \mathrm{~cm}$.
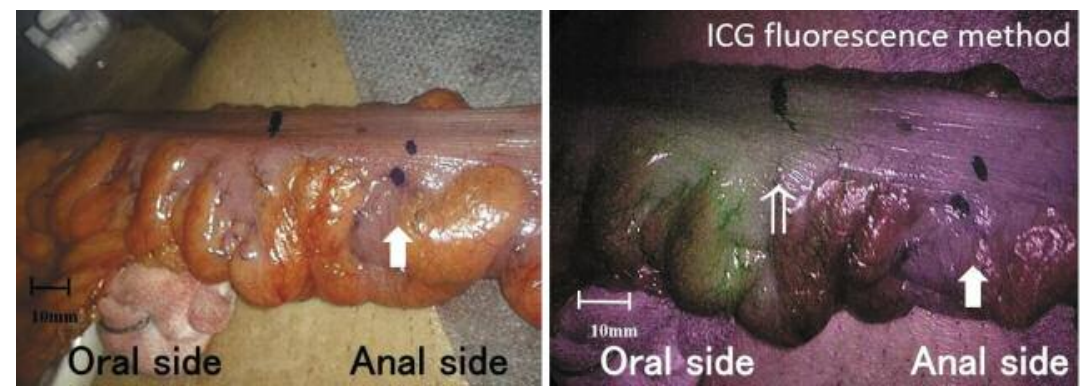

Figure 3. The patient in this case was a 71-year-old man with lower rectal cancer after preoperative chemoradiotherapy. The white arrow indicates the oral-side intestinal transection line at first judgment. Based on the indocyanine green (ICG) fluorescence method, the intestinal transection line was changed to the position indicated by the open white arrow. The distance between positions was $3 \mathrm{~cm}$.
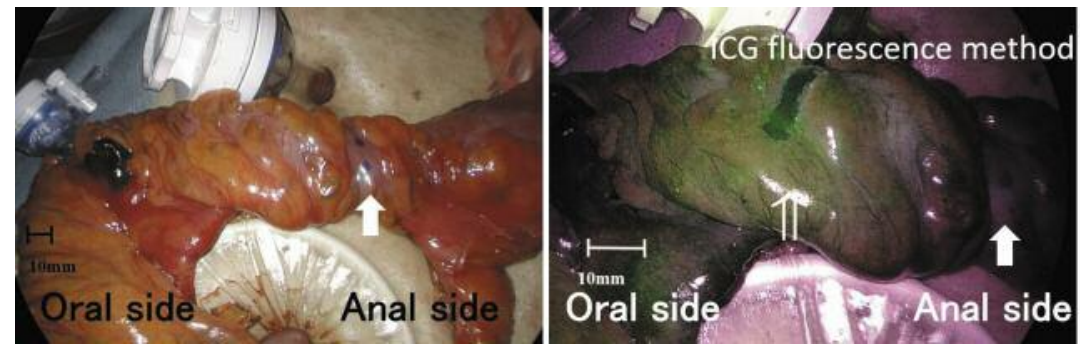

Figure 4. The patient in this case was a 64-year-old man with lower rectal cancer. The white arrow indicates the oral-side intestinal transection line at first judgment. The intestinal transection line was changed to the position indicated by the open white arrow after use of the indocyanine green (ICG) method. The distance between these positions was $4 \mathrm{~cm}$. 
Table II. Operative procedures used in this study.

\begin{tabular}{lc}
\hline Procedure & Frequency, n (\%) \\
\hline Ileocecal resection & $11(22 \%)$ \\
Right hemicolectomy & $10(20 \%)$ \\
Sigmoidectomy & $6(12 \%)$ \\
High-anterior resection & $4(8 \%)$ \\
Low-anterior resection & $10(20 \%)$ \\
Super low-anterior resection & $4(8 \%)$ \\
Partial resection & $1(2 \%)$ \\
Intersphincteric resection & $1(2 \%)$ \\
Miles' operation & $1(2 \%)$ \\
Hartmann operation & $2(4 \%)$ \\
Covering ileostomy & $10(20 \%)$ \\
Changed intestinal transection line & $3(6 \%)$ \\
\hline
\end{tabular}

( $\mathrm{n}=139$, left hemicolectomy, anterior resection). In addition, the surgical plan was changed for $7.9 \%$ of patients such as changes to the intestinal resection line. No postoperative anastomotic leakage was observed in those cases (5). Kudszus et al. (6) also showed that the IBF in the planned anastomosis was insufficient in $13.9 \%(28 / 201)$ of patients with colorectal cancer using the ICG fluorescence method.

We also encountered three cases $(6 \%)$ in which changes to the transection line with the ICG fluorescence method were made. When limiting the sample to rectal cancers alone, the frequency of changing the transection line was $14.2 \%(3 / 21)$. One of these cases was a very high-risk case for anastomotic leakage, a male patient with lower rectal cancer, lymph node metastasis and preoperative radiochemotherapy (Figure 3). Despite this concerning background, no postoperative complications were encountered. Anastomotic leakage may occur in such a case if the intestinal resection line for anastomosis had not been changed using the ICG fluorescence method. One patient who actually experienced anastomotic leakage was judged to have no problem from the IBF evaluation using the ICG fluorescence method. This was a case after preoperative management of fasting with preoperative bowel obstruction. The cause of anastomotic leakage in this case was suspected to be obstructive colitis.

Because anastomotic leakage can arise as a result of multiple factors, ensuring there is no anastomotic leakage is difficult. However, the ICG fluorescence method may lower the influence of blood flow as a risk factor. Wada et al. reported a quantitative blood flow evaluation study for anastomosis of left-sided colorectal cancer using an external fluorescent camera (PDE-neo System; Hamamatsu Photonics, Hamamatsu, Japan) (13). Their retrospective study used stored operative movie and ROI analysis software (Hamamatsu Photonics) to analyze fluorescence intensity, and the difference in fluorescence between maximum and baseline $\left(\mathrm{F}_{\max }\right)$ and the time from first fluorescence increase to maximum $\left(\mathrm{T}_{\max }\right)$
Table III. Postoperative complications.

\begin{tabular}{lcc}
\hline Complication & $\mathrm{n}(\%)$ & $\begin{array}{c}\text { Clavien-Dindo grade III } \\
\text { or more, } \mathrm{n}(\%)\end{array}$ \\
\hline Anastomotic leakage & $1(2 \%)$ & $1(2 \%)$ \\
Paralytic ileus & $1(2 \%)$ & 0 \\
Abdominal abscess & $1(2 \%)$ & 0 \\
Stomal obstruction & $1(2 \%)$ & 0 \\
Chylous ascites & $2(4 \%)$ & 0 \\
Wound infection & $2(4 \%)$ & 0 \\
\hline
\end{tabular}

were defined from ROI analyses. As a result, $\mathrm{F}_{\max }$ and $\mathrm{F}_{\max } / \mathrm{T}_{\max }$ were found to be predictive of anastomotic leakage. Their results show the possibility of making the ICG fluorescence method more objective. However, their method is not simple and does not provide real-time feedback for selecting anastomotic sites during surgery nor for determining whether surgical procedures need to be changed.

For future prospects, the means of making the ICG fluorescence method more objective and of providing feedback for intraoperative judgments are anticipated. Key limitations of this study were the retrospective design, the small number of patients from a single institution and the possibility of selection bias.

\section{Conflicts of Interest}

The Authors declare that they have no competing interests in regard to this study.

\section{Authors' Contributions}

SR made substantial contributions to conception and design, acquisition of data, analysis and interpretation of data. KS, TK, MI, TU, TO and KE discussed this study. KY gave final approval of the version to be submitted. All Authors read and approved the final article.

\section{Acknowledgements}

The Authors would like to thank professor Masashi Yoshida (Department of Surgery, International University of Health and Welfare Hospital) for his invaluable advice on the ICG fluorescence method, and the medical staff at Jikei University Daisan Hospital.

This work was supported by JSPS KAKENHI Grant Number 19K20735.

\section{References}

1 Ozawa Y, Murakami M, Watanabe M, Yoshizawa S, Goto S, Otsuka $\mathrm{K}$ and Aoki T: Preoperative colonic cancer tattooing using the near-infrared fluorescence laparoscopic imaging system. Asian J Endosc Surg 9: 340-343, 2016. PMID: 27790874. DOI: $10.1111 /$ ases.12306 
2 Ris F, Hompes R, Cunningham C, Lindsey I, Guy R, Jones O, George B, Cahill RA, and Mortensen NJ: Near-infrared (NIR) perfusion angiography in minimally invasive colorectal surgery. Surg Endosc 28: 2221-2226, 2014. PMID: 24566744. DOI: 10.1007/s00464-014-3432-y

3 Jafari MD, Lee KH, Halabi WJ, Mills SD, Carmichael JC, Stamos MJ and Pigazzi A: The use of indocyanine green fluorescence to assess anastomotic perfusion during robotic assisted laparoscopic rectal surgery. Surg Endosc 27: 3003-3008, 2013. PMID: 23404152. DOI: 10.1007/s00464-013-2832-8

4 Sherwinter DA: Transanal near-infrared imaging of colorectal anastomotic perfusion. Surg Laparosc Endosc Percutan Tech 22: 433-436, 2012. PMID: 23047388. DOI: 10.1097/SLE. 0b013e3182601eb8

5 Jafari MD, Wexner SD, Martz JE, McLemore EC, Margolin DA, Sherwinter DA, Lee SW, Senagore AJ, Phelan MJ and Stamos MJ: Perfusion assessment in laparoscopic left sided/anterior resection (PILLAR) II: A multi-institutional study. J Am Coll Surg 220: 82-92, 2015. PMID: 25451666. DOI: 10.1016/ j.jamcollsurg.2014.09.0155.

6 Kudszus S, Roesel C, Schachtrupp A and Höer JJ: Intraoperative laser fluorescence angiography in colorectal surgery: A noninvasive analysis to reduce the rate of anastomotic leakage. Langenbecks Arch Surg 395: 1025-1030, 2010. PMID: 20700603. DOI: $10.1007 / \mathrm{s} 00423-010-0699-\mathrm{x}$

7 Watanabe J, Ota M, Suwa Y, Ishibe A, Masui H and Nagahori $\mathrm{K}$ : Real-time indocyanine green fluorescence imaging-guided complete mesocolic excision in laparoscopic flexural colon cancer surgery. Dis Colon Rectum 59: 701-705, 2016. PMID: 27270525. DOI: 10.1097/DCR.0000000000000608
8 Japanese Society for Cancer of the Colon and Rectum: Japanese Classification of Colorectal Carcinoma (English ed 2). Kanehara, Tokyo, 2009.

9 Dindo D, Demartines N, and Clavien PA: Classification of surgical complications: a new proposal with evaluation in a cohort of 6336 patients and results of a survey. Ann Surg 240: 205-213, 2004. PMID: 15273542. DOI: 10.1097/01.sla.0000133083.54934.ae

10. Law Wl, Chu KW, Ho JW, and Chan CW: Risk factors for anastomotic leakage after low anterior resection with total mesorectal excision. Am J Surg 179: 92-96, 2000. PMID: 10773140. DOI: $10.1016 / \mathrm{s} 0002-9610$ (00)00252-x

11 Kang CY, Halabi WJ, Chaudhry OO, Nguyen V, Pigazzi A, Carmichael JC, Mills S, and Stamos MJ: Risk factors for anastomotic leakage after anterior resection for rectal cancer. JAMA Surg 148: 65-71, 2013. PMID: 22986932. DOI: 10.1001/2013.jamasurg.2

12 Altın O and Alkan M: Risk factors associated with anastomotic leakage in patients operated due to colorectal tumours. Med Glas (Zenica) 16: 1013-1019, 2019. PMID: 31077126. DOI: 10.17392/1013-19

13 Wada T, Kawada K, Takahashi R, Yoshitomi M, Hida K, Hasegawa $S$ and Sakai Y: ICG fluorescence imaging for quantitative evaluation of colonic perfusion in laparoscopic colorectal surgery. Surg Endosc 31: 4184-4193, 2017. PMID: 28281123. DOI: $10.1007 / \mathrm{s} 00464-017-5475-3$

Received November 27, 2019

Revised December 4, 2019

Accepted December 6, 2019 